\title{
Tecnología y transformación de la práctica docente en la formación en Comunicación en la era 2.0: un caso de estudio
}

\author{
Josep Maria Blanco I PonT \\ Universitat Autònoma de Barcelona (UAB) \\ josepmaria.blanco@uab.cat \\ Patrícia LÁzARo PERNIAS \\ Universitat Autònoma de Barcelona (UAB) \\ patricia.lazaro@uab.cat
}

Recibido: $17 / 10 / 2012$

Aceptado: 23/01/2013

\begin{abstract}
Resumen
Las NTIC han transformado la práctica formativa universitaria de un modo radical. En el caso de los docentes cuyas materias se orientan a la elaboración de productos comunicativos profesionales, y que deben emplear otras tecnologías, la complejidad es aún mayor. En el trabajo que se presenta se ofrecen las aportaciones de la primera fase, de exploración cualitativa, de una investigación diseñada para conocer las transformaciones que este perfil de profesor ha sufrido con el paso del tiempo. También se presentan valoraciones generales sobre el enfoque de esta investigación que están desarrollando miembros del Grupo de Investigación en Publicidad y Relaciones Públicas (GRP) de la Universidad Autónoma de Barcelona, que lo aplicarán al caso de la Facultad de Ciencias de la Comunicación de dicha universidad.
\end{abstract}

Palabras clave: Universidad, Docencia, Tecnología, Formación, Perfil.

\section{Technology and Transformation of Teaching Practice in Training in Communication in the 2.0 Era: a Case Study}

\begin{abstract}
The ICTs has transformed the university's teaching practice in a radical way. The difficulty can be greater for university professors that use technologies to explain the professional production of communication contents. The study explores the profile transformations of these professors over time. We provide preliminary conclusions of qualitative research applied to the case of the Faculty of Communication Sciences at the Autonomous University of Barcelona where the authors are teachers and members of the Research Group in Advertising and Public Relations (GRP).
\end{abstract}

Keywords: University, Teaching, Technology, Training, Profile.

\section{Referencia normalizada}

BLANCO I PONT, Josep Maria y LÁZARO PERNIAS, Patrícia (2013): “Tecnología y transformación de la práctica docente en la formación en Comunicación en la era 2.0: un caso de estudio". Estudios sobre el mensaje periodístico. Vol. 19, Núm. especial abril, págs.: 623-631. Madrid, Servicio de Publicaciones de la Universidad Complutense.

Sumario: 1. Introducción. 2. Metodología. 3. Desarrollo; 3.1 Etapa inicial: estudio contextual y entrevistas en profundidad; 3.2. Aportaciones de las entrevistas en profundidad. 4. Conclusiones; 4.1. Conclusiones metodológicas preliminares. 5. Referencias bibliográficas

\section{Introducción}

Las tecnologías de la información y la comunicación, y sobre todo la conversión de la información en dígitos transportables a través de medios y señales de diferentes ca- 
racterísticas, han transformado en apenas 30 años la manera de convivir en el planeta de un modo radical. La revolución digital ha cambiado nuestras vidas sin apenas haber tenido tiempo de asumir esa mutación. Por supuesto, este tsunami ha pasado por encima de todos aquellos que emplean herramientas de la comunicación para aprender y para ayudar a aprender. De modo que las tareas que se exigen al docente universitario evolucionan para adaptarse a los cambios sobre la manera de entender la transmisión de conocimiento que la comunidad educativa propone.

Esta mutación drástica y sutil ha obligado a los profesores a olvidar posturas apocalípticas si de verdad quieren continuar realizando su tarea sin quedarse totalmente alejados de la forma como sus discentes tienen de aprehender el mundo y construir su realidad. En el caso de aquellos que emplean las tecnologías de la comunicación para estudiar y desarrollar sus usos comunicativos, la complejidad puede resultar aún mayor, puesto que no basta con conocer las que usan los medios de comunicación sino que también debe mostrarse una actitud permeable a la comprensión, aunque sea generalizada, de aplicaciones y software que pueden utilizarse para generar y compartir información desde cualquier dispositivo móvil capaz de conectarse a una red.

Pero, ¿de qué modo el docente ocupado en conocer y utilizar tecnologías y software para poder emplearlas de modo profesional para comunicar ha visto su vida "alterada" o ha "asumido" esas obligaciones y responsabilidad a lo largo de este tiempo? Interesados por este asunto se ha puesto en marcha un estudio, en fase inicial, que pretende ver de qué modo esos cambios han modelado el perfil del nuevo docente de la era 2.0.

\section{Metodología}

El Espacio Europeo de Educación Superior ha supuesto un cambio en cuanto a organización universitaria y también ha pretendido ser el marco para una transformación profunda de las formas pedagógicas en la enseñanza superior. Así, las nuevas metodologías docentes se han planteado un profesor que debe dejar de ser exclusivamente un vehículo de transmisión de información a los estudiantes, para convertirse en un facilitador del proceso de aprendizaje y un orientador en la búsqueda y uso de la información por parte del alumno. A su vez, el estudiante tiene que abandonar su papel pasivo, de receptor de esa información, para convertirse en un agente activo, constructor y protagonista de su propio proceso formativo (Lázaro Lorente, 1993: 19; Fumat, 1991: 201; Tourón, Altarejos, Reparaz, 1991: 381).

Se supone que el docente no es sólo un profesional con gran dominio de su área de conocimiento, sino que también ha adquirido nuevas competencias que le permiten, entre otros muchos objetivos, acotar con claridad el plan formativo de los estudiantes y prepararlos para la inserción en el entorno laboral. Además, va a ser imprescindible que sea capaz de seleccionar las metodologías más apropiadas para cada momento del proceso de aprendizaje. Así, junto a las clásicas sesiones expositivas, orientadas a la transmisión de información, se imponen otras formas de docencia que favorecen un trabajo directo con los estudiantes. Es el caso de seminarios, tutorías o prácticas de laboratorio, para resolución conjunta de problemas o seguimiento personalizado del trabajo y logros de los estudiantes. Y se deberá progresar en las pro- 
puestas encaminadas a favorecer e incrementar el trabajo autónomo del estudiante. Todo ello en un momento en el que la docencia a grandes grupos no ha desaparecido y debe convivir con otra más personalizada ${ }^{1}$.

En este contexto, el docente necesitará desplegar competencias comunicativas y de interacción con los estudiantes, tanto a nivel grupal como individual. De entre estas competencias cabe destacar las habilidades comunicativas (saber expresarse, hablar en público), para hacerse comprender por los estudiantes. También es importante saber crear un clima en el proceso de aprendizaje: "La idea de "clima" recoge un conjunto de aspectos que, se supone, condicionan la calidad del funcionamiento del aula. (...) De alguna manera son condiciones que caracterizan el trabajo en el aula y que afectan de manera fundamental al nivel de satisfacción que podamos sentir en nuestro trabajo" (Zabalza, 2006: 124). Y, en especial, potenciar las habilidades en las relaciones interpersonales (Zabalza, 2006: 125).

Otras capacidades del docente EEES pasan por el conocimiento y dominio de aquellas herramientas que mejoren el proceso de enseñanza/aprendizaje. Así, saber manejarse con apoyo NTIC para la preparación y uso de materiales didácticos que faciliten la transmisión de información y la comprensión de contenidos y aprovechamiento de instrumentos que activen la interacción con el estudiante: correo electrónico, foros de discusión, redes sociales. Y en el caso de las asignaturas de comunicación, el conocimiento de las NTIC como objeto de estudio propio.

Es casi obligado para el buen profesor el dominio de las TIC. En este sentido, existe una variada bibliografía en la que se analiza su papel en el entorno educativo universitario (Hernández et alt. 2011; Sangrà y Sanmamed, 2004; Sierra y Sotelo, 2010). Aunque las brechas tecnológicas se generan en todos los niveles, incluidos los dedicados a la transferencia de conocimiento de nivel superior. Los docentes que se formaron en la época "analógica" se ven obligados a realizar un esfuerzo mayor en la asunción de nuevas tecnologías para ayudar en el aprendizaje a estudiantes que son nativos digitales. Ya en el 2008, la Unesco advertía de la necesidad de dotar de herramientas y capacidades a docentes para asumir los retos que presenta este nuevo horizonte:

"Today's classroom teachers need to be prepared to provide technology-supported learning opportunities for their students. Being prepared to use technology and knowing how that technology can support student learning have become integral skills in every teacher's professional repertoire. Teachers need to be prepared to empower students with the advantages technology can bring. Schools and classrooms, both real and virtual, must have teachers who are equipped with technology resources and skills and who can effectively teach the necessary subject matter content while incorporating technology concepts and skills. Interactive computer simulations, digital and open educational resources, and so-

${ }^{1}$ El espíritu "Bolonia", de trabajo personalizado y en grupos pequeños, ha sido desvirtuado con el incremento del número de matrícula. Así, en las licenciaturas impartidas por la Facultad de Ciencias de la Comunicación de la UAB, la matrícula se situaba en 80 personas para grupos teóricos, que solían subdividirse en tres grupos prácticos. Con el nuevo grado, la matrícula se ha elevado a unos 140 estudiantes en sesiones teóricas. 
phisticated data-gathering and analysis tools are only a few of the resources that enable teachers to provide previously unimaginable opportunities for conceptual understanding".

"Traditional educational practices no longer provide prospective teachers with all the necessary skills for teaching students to survive economically in today's workplace". (UNESCO, 2008: 1)

Aunque este marco es válido para maestros y profesores en general, creemos que no se ha estudiado suficientemente el papel del docente cuando debe compatibilizar su conocimiento de las NTIC con las tecnologías propias de la Comunicación Social Profesional. Este trabajo pretende dar un paso en esa dirección.

Con el fin de comprobar el alcance de los cambios que los planteamientos pedagógicos y las transformaciones tecnológicas del entorno provocan en la práctica del profesor universitario, nos planteamos responder a diferentes objetivos:

» Determinar si existe un nuevo perfil de docente con nuevas habilidades en el uso de las herramientas NTIC.

» Estudiar las características de ese nuevo profesorado.

»Valorar el equilibrio entre las oportunidades formativas en TIC que la estructura universitaria pone a su disposición y el uso de esas tecnologías en el trabajo diario.

»Valorar el equilibrio entre la disposición del profesorado al aprendizaje de nuevas competencias (uso de diferentes metodologías docentes, TIC) y su distribución de tiempos laborales y personales.

\section{Desarrollo}

\subsection{Etapa inicial: estudio contextual y entrevistas en profundidad}

Tras el análisis documental de fuentes que nos sirvan para contextualizar la evolución en la asunción del compromiso educativo por parte de ese docente en concreto -extrapolables muchas de las informaciones al resto de la comunidad científica y docente de la universidad española-, hemos puesto en marcha una selección preliminar de individuos a quienes realizar entrevistas en profundidad. Estos primeros profesores comparten una serie de requisitos, de entre los que destaca el uso continuado de tecnologías empleadas para la práctica profesional comunicativa así como de TIC en la práctica docente durante los últimos 15 años.

Las primeras entrevistas en profundidad se completarán con otras nuevas a perfiles similares, adaptando la técnica de bola de nieve a partir de los primeros análisis, hasta obtener una saturación de las unidades de información. Posteriormente esas unidades tendrán una traducción en variables para la elaboración de un cuestionario. Esta encuesta será lanzada a muestras de docentes divididas en estratos cuyas características deben determinarse luego de la etapa en curso.

Uno de los objetivos del artículo es la discusión sobre la metodología empleada y el diseño de protocolo para llevar a cabo la investigación, para poderlos modelar de acuerdo al estudio de situaciones en otras facultades, otras universidades e, incluso, en otros ámbitos.

Este último apunte nos permite aprovechar la circunstancia para incidir en la importancia de observar el contexto en el que se produce el uso y empleo de tecnologías 
tanto para la formación en general, como para la formación de estudiantes que van a tener que emplear esas herramientas de modo profesional.

A modo de ejemplo, y puesto que llevaremos a cabo el análisis de un caso concreto -el de la Facultad de Ciencias de la Comunicación de la UAB-, apuntaremos que en la delimitación de los períodos de estudio cabe tener en cuenta cuestiones tales como el diseño y implementación de planes de estudios (influidos a su vez por la teoría pedagógica en las que se basa su desarrollo), cambios producidos por el paso de una época tecnológica anterior a otra nueva y factores como las competencias y habilidades de los estudiantes en el uso de NTIC de comunicación profesional. En estos cambios, la accesibilidad y generalización del uso de software en distintos dispositivos ha jugado un papel determinante.

Para ilustrar a lo que nos referimos, realizaremos un breve resumen de las generalidades observadas y los períodos que pueden distinguirse en el estudio de la facultad mencionada. El análisis exhaustivo que llevamos a cabo en el trabajo -en el que completamos el análisis documental con testimonios de personas protagonistas de las decisiones sobre adopción de tecnologías-, nos ayuda en la preparación de los cuestionarios y en la interpretación de los mismos.

Durante la era analógica del centro (los setenta y parte de los ochenta), la docencia debía realizarse de forma presencial, y la máquina de escribir (que ve como aparecen los primeros ordenadores con floppies), es la protagonista de la mayoría de documentos docentes. La maquinaria de producción de contenidos comunicativos profesionales resultaba voluminosa, cara y, en general, escasa en cuanto a acceso y uso (siempre mediado técnicamente). La organización resultaba, pues, complicada, puesto que había pocos equipos y pocos espacios habilitados para la simulación de prácticas profesionales.

En la era digital, (años ochenta y noventa) y con una facultad pensada para la docencia en Ciencias de la Comunicación, la aparición de los primeros ordenadores de sobremesa impulsa cambios en la organización administrativa y también en la docente. El correo electrónico transformará las dinámicas de coordinación y la realización de prácticas y de disponibilidad y creación de recursos para los estudiantes. Empieza a intuirse el alcance del teletrabajo y ya desde 1996 se cuenta con un "Campus Virtual" en el que se promueve la gestión de los grupos de estudiantes y de los materiales docentes. El centro apuesta por diferentes tipos de hardware y de software para la gestión de archivos de distintos formatos y contenidos. Se incrementan la maniobrabilidad de equipos de producción y la adecuación de espacios para la realización de prácticas. La mediación técnica disminuye. Los estudiantes mejoran su organización al promoverse una mayor descentralización de la preproducción. Las tecnologías de consumo doméstico indican lo que será ya una tendencia irreversible hacia la adopción de formatos digitales y el abandono, en la siguiente etapa, de la filosofía de producción analógica.

En la era 2.0, iniciada prácticamente con el cambio de siglo, el anclaje a un único lugar de producción de contenidos pasa a la historia. El teletrabajo amplia el tiempo de dedicación a las diferentes tareas docentes a toda la jornada. También la atención virtual cambia las lógicas de relación anteriores y busca el ajuste con un calculado contacto presencial. Se trata de una etapa caracterizada por la profusión de software, 
aplicaciones y la hegemonía de los buscadores. Los docentes y estudiantes comparten ahora la posibilidad de ser gestores y productores de contenidos. La complejidad de abordar el análisis del uso que hace la sociedad de los instrumentos tecnológicos y la aparición de las redes sociales influye en el perfil del docente. Éste se siente obligado a observar el comportamiento de las mismas a través del uso de dispositivos o aplicaciones, puesto que debe transmitir la complejidad de la tarea del comunicador profesional en un momento en que cada persona es una productora de información y contenidos en potencia. Todo esto en plena crisis, con efectos claros sobre el cuerpo docente del centro y de la universidad en general, que debe someter a dieta a las plantillas de los departamentos. La disminución de la contratación de profesores asociados es uno de los daños colaterales más evidentes. Los docentes supervivientes deben hacer más con menos. De las 37,5 horas semanales que debe dedicar a su labor docente un profesor titular, 12 deben estar destinadas a la docencia y 6 a la atención de alumnos (según el Reglament de Personal Acadèmic de la UAB, aprobado en Consejo de Gobierno, 25/4/2012). Esta propuesta convierte en un sudoku de dimensiones extraordinarias el cálculo del plan docente, cuando se diseccionan todas y cada una de las tareas que deben hacerse en las distintas asignaturas de grado y de máster.

En cuanto al tiempo restante disponible, debemos contabilizar las labores relacionadas con la investigación, con el reciclaje continuado en métodos docentes en el marco del EEES y el conocimiento de las tecnologías empleadas para la gestión de contenidos y la formación en Comunicación. También el tiempo dedicado a conocer los usos de las tecnologías que los públicos emplean para estar informados o para cualquier otra actividad y pensar cómo usarlas como herramientas para la enseñanzaaprendizaje, saltando en ocasiones el marco institucional universitario y aprovechando los recursos de la red ${ }^{2}$. Se constata una ausencia del cálculo del tiempo no dedicado específicamente a la labor con alumnos o a la investigación; el tiempo que el docente necesita para hacer compatible las actividades laborales con su vida personal. Descubrir el papel que juega la tecnología en esa dificultad y esfuerzo personal que hay que realizar para encontrar espacios personales, es uno de los objetivos de este trabajo.

\subsection{Aportaciones de las entrevistas en profundidad}

Aunque pueda resultar prematuro, ya que, como hemos indicado, no daremos por completa la primera fase de entrevistas en profundidad hasta haber saturado las unidades de información, creemos que podemos aportar algunos apuntes útiles:

Según varios de los primeros entrevistados, la sociedad no tiene una idea clara de la labor del docente, así como del ámbito concreto de la formación en Comunicación.

A pesar de que emplea las tecnologías en el desempeño de su tarea como profesor, es consciente que debe hacer un esfuerzo para transmitir a los alumnos que ese no es conocimiento suficiente, sino que es fundamental la formación en lenguajes comuni-

2 Las redes sociales y los dispositivos móviles plantean nuevos retos para la relación entre universidad y sociedad en red. Recomendamos la lectura del trabajo La Universidad en la sociedad Red de DUART et alt. (2008), en el que se estudian los usos de internet en Educación Superior. 
cativos y las habilidades que permiten al comunicador compatibilizar sus conocimientos sobre comunicación y las herramientas.

El docente que emplea NTIC para la docencia y para la formación en comunicación asume el esfuerzo por estar pendiente de las novedades y de su propio reciclaje, tanto a nivel pedagógico como profesional, independientemente de los cursos que se ofrezcan por parte de la universidad o el centro.

El software libre y las aplicaciones empleadas en redes sociales no pueden eludirse por completo y deben encajarse en la tarea formativa de la materia. Con todo, debe existir un alto grado de control sobre esas implementaciones, de acuerdo con los objetivos del curso, ya que la organización puede verse alterada provocar ruido entre el equipo docente y el grupo clase.

En la actualidad, a algunos de los profesores no les parece diáfano el cálculo real de su esfuerzo, ni interpretan con claridad la tarea de los responsables de la asignación docente. Se cree que el problema es fruto del proceso de implantación del EEES y que acabará por mejorar. Son conscientes que el teletrabajo complica el cálculo de su dedicación, ya que ésta acaba por dilatarse a lo largo de las 24 horas del día, en una continua decisión personal de asignar tiempos a tareas personales o laborales.

El concepto tiempo de descanso se identifica con dormir, a lo que se destina entre $7 \mathrm{u} 8$ horas diarias. Las actividades de tiempo libre son parte del tiempo de descanso. Durante el fin de semana puede dedicarse un tiempo a la revisión de trabajos de los alumnos, preparación de materiales docentes, corregir, evaluar y obtener resultados que deban ser consultados al inicio de la semana siguiente, en especial en ciertos períodos del curso. La resolución de dudas de los estudiantes se deja, la mayor parte de las veces, para los días laborables.

\section{Conclusiones}

En términos generales, creemos necesario emprender un análisis en profundidad de la autoimagen que tienen los docentes universitarios de su trabajo y la valoración que del mismo se hace tanto en la esfera profesional como social. Por lo que se refiere al ámbito concreto en el que llevamos a cabo el estudio, consideramos que esta investigación puede aportar interesantes resultados sobre el impacto del uso de NTIC para el apoyo docente y del empleo de tecnologías para la comunicación profesional en la docencia universitaria de graduados. El desempeño como profesor universitario obliga, además, a prestar atención al cambio experimentado en el seno de la sociedad a causa del uso de tecnologías de la comunicación y redes sociales y, sobre todo entre los propios estudiantes interesados en ejercer como expertos y profesionales de la Comunicación. Así será posible avanzar en el diseño de dinámicas que puedan ser incorporadas en las asignaturas, de acuerdo con los requerimientos del EEES.

\subsection{Conclusiones metodológicas preliminares}

A nivel metodológico, en cuanto a nuevas entrevistas y futuros cuestionarios, es importante discernir entre la realidad de la práctica y la percepción que el docente tiene sobre su responsabilidad, su perfil, su dedicación y su conocimiento de las herramientas tecnológicas. Este matiz debe tener una traducción en la formulación de las preguntas y también en la identificación y desarrollo del concepto que nos permita 
estudiar el fenómeno. Creemos necesario, elaborar un constructo conceptual específico para estudiar la interpretación que los profesores realizan de la relación subjetiva entre el grado de cumplimiento de responsabilidades docentes e investigadoras (asumidas personalmente y/o asignadas) y las facilidades y dificultades encontradas en el entorno personal y profesional para poder asumirlo.

En cualquier caso, y en el asunto que nos ocupa -en el que estudiamos las opiniones de profesores que, simultáneamente, emplean NTIC para su docencia y explican el uso de las tecnologías de la práctica de la Comunicación profesional-, parece adecuado seguir preguntando acerca de la percepción del entrevistado sobre la evolución sufrida por ambos tipos de tecnologías. Debe realizarse un esfuerzo para que el docente explique el matiz entre el uso de la herramienta comunicativa ya sea con objetivos docentes o ya sea para ayudar en su desempeño en el uso profesional.

Por lo que se refiere al diseño de asignaturas, es importante incidir en cuáles son los factores que llevan a decidir la integración de un tipo u otro de tecnologías y software. También debe preguntarse sobre si en ese tipo de resoluciones intervienen los cambios detectados en cuanto a conocimientos y habilidades de los estudiantes en el uso de los mismos. Una dificultad añadida en el momento actual constituye en esforzarse en disipar la confusión que puede producirse entre conceptos tales como software y tecnología usada para comunicar, o entre red social y aplicación, confusiones que la propia industria parece que está muy interesada en no resolver.

Parece evidente preguntar sobre las transformaciones que el docente cree que ha tenido que realizar para ajustar sus características personales y el modo de entender su tarea como docente e investigador, con los requerimientos y métodos pedagógicos promocionados en la aplicación de los diferentes planes de estudio. Y puesto que puede tener diferentes tipos y grados de "gratificaciones" de acuerdo a su compromiso con la tarea de docente e investigador de una universidad pública, es importante preguntar explícitamente si la relación entre el trabajo realizado y la retribución económica percibida es justa.

Finalmente, parece necesario afinar en aquellas preguntas que permitan conocer con mayor detalle las formas y modos como los docentes transforman sus agendas y horarios para poder compatibilizar sus obligaciones y el nivel de compromiso con sus labores profesionales (formación y reciclaje) con la conciliación con la vida personal y la vida familiar. Las diferentes maneras como los individuos pueden usar el tiempo y las tecnologías disponibles en el espacio que emplean como residencia deben tenerse en cuenta. Sobre todo en casos en los que una cierta rutina o la dinámica habitual se ven interrumpidas, por ejemplo, por cuestiones derivadas de estar al cargo de otros, asistir a actividades profesionales, congresos, conferencias, o actos institucionales, o cuando no hay más remedio que tener que superar una lesión física o convivir con una enfermedad.

\section{Referencias bibliográficas}

DUART, Josep Maria, GIL, Marc, PUJOL, Maria, y CASTAÑO, Jonatan (2008): La Universidad en la sociedad Red. Usos de internet en Educación Superior. Barcelona, Ariel. 
FUMAT, Yveline (1991): “Enseignement à distance. Enseignement présentiel”, en La pedagogia universitària. Un repte a l'ensenyament superior, Barcelona, Divisió de Ciències de l'Educació, pp. 201-205.

HERNÁNDEZ, José, PENNESI, Massimo, SOBRINO, Diego, VÁZQUEZ, Azucena (2011): Informe Experiencias educativas en las aulas del siglo XXI. Innovación con TIC., Madrid, Ariel.

LÁZARO LORENTE, Luis Miguel, (ed., 1993): Formación pedagógica del profesorado universitario y calidad de la educación, Valencia, Formació Permanent de la Universitat de València y Ministerio de Educación y Ciencia.

SANGRÀ, Albert, GONZALEZ SANMAMED, Mercedes (coord., 2004): La transformación de las universidades a través de las TIC: discursos y prácticas. Barcelona, Editorial UOC.

SIERRA, Javier, SOTELO, Joaquín, (coords., 2010): Métodos de innovación docente aplicados a los estudios de Ciencias de la Comunicación. Madrid, Editorial Fragua.

TOURÓN, Javier; ALTAREJOS, Francisco; REPARAZ, María Rosario (1991): "Los roles del profesor y del alumno en la Enseñanza Universitaria", en La pedagogia universitària. Un repte a l'ensenyament superior. Barcelona, Divisió de Ciències de l'Educació, pp. 381-387.

UNESCO (2008): "UNESCO's project «ICT Competency Standards for Teachers»", http://unesdoc.unesco.org/images/0015/001562/156207e.pdf. [fecha de consulta: 24 de marzo de 2012]

ZABALZA, Miguel Ángel (2006): Competencias docentes del profesorado universitario. Calidad y desarrollo profesional. Madrid, Editorial Narcea.

Josep Maria BLANCO I PONT

Universitat Autònoma de Barcelona

Departament de Publicitat. Relacions Públiques i Comunicació Audiovisual

Profesor Titular de Universidad

josepmaria.blanco@uab.cat

\section{Patrícia LÁZARO PERNIAS}

Universitat Autònoma de Barcelona

Departament de Publicitat. Relacions Públiques i Comunicació Audiovisual

Profesora Titular de Universidad

patricia.lazaro@uab.cat 